\title{
À la recherche d'un cours monétaire canadien : 1760-1777
}

\section{Jean Hamelin}

Volume 15, numéro 1, juin 1961

URI : https://id.erudit.org/iderudit/302092ar

DOI : https://doi.org/10.7202/302092ar

Aller au sommaire du numéro

Éditeur(s)

Institut d'histoire de l'Amérique française

ISSN

0035-2357 (imprimé)

1492-1383 (numérique)

Découvrir la revue

Citer cet article

Hamelin, J. (1961). À la recherche d'un cours monétaire canadien : 1760-1777.

Revue d'histoire de l'Amérique française, 15(1), 24-34.

https://doi.org/10.7202/302092ar d'utilisation que vous pouvez consulter en ligne.

https://apropos.erudit.org/fr/usagers/politique-dutilisation/ 


\section{À LA RECHERCHE D'UN COURS MONÉTAIRE CANADIEN : $1760-1777$}

Qui veut démêler la situation monétaire si confuse dans les trois gouvernements de la province de Québec sous le régime militaire doit d'abord définir le système monétaire en vigueur dans les métropoles européennes. Ce système a été maintes fois décrit par les historiens et les économistes qui l'ont baptisé le système des étalons parallèles. ${ }^{1}$ On pourrait résumer de la sorte ses caractéristiques:

a) Les pièces métalliques en circulation renferment un certain nombre de grammes d'or ou d'argent. ${ }^{2}$ C'est le poids de la monnaie. Le poids fonde la valeur intrinsèque de la monnaie. Si la monnaie d'or ou d'argent possède une valeur intrinsèque qui correspond au cours commercial des métaux précieux, la monnaie de cuivre et de billon n'est qu'un jeton sans valeur en soi.

b) Une monnaie souvent imaginaire, dite monnaie de compte, sert de barème aux monnaies réelles. Son nom varie suivant les pays: on l'appelle livre tournois en France et livre sterling en Angleterre; douze pence font un chelin, vingt chelins font une livre tournois. Des ordonnances royales fixent la valeur de chaque pièce de monnaie en circulation par rapport à la monnaie de compte. Ainsi le roi de France peut décréter que l'écu d'or soleil - une monnaie réelle - vaut 20 livres tournois et que l'écu d'argent en vaut six. La valeur en monnaie de compte de chaque pièce de monnaie ainsi fixée par ordonnance s'appelle

$1 \mathrm{~B}$. Nogaro, La Monnaie et les systèmes monétaires, 73ss.

2 On pèse les monnaies suivant le système de Troyes:

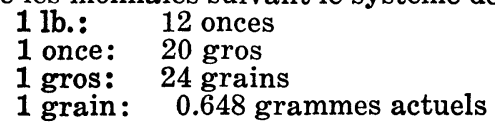


la valeur nominale. Le lien que les ordonnances établissent entre la monnaie réelle et la monnaie de compte n'a rien de permanent et le roi peut le modifier à volonté.

c) La relation entre la valeur nominale des pièces d'or et d'argent s'établit, en principe, non pas par les cours atteints par ces métaux sur les marchés commerciaux, mais par la monnaie de compte. Ce n'est qu'une fois que le roi a décrété qu'une pièce contenant 15 grains d'argent ou 1 grain d'or vaut 1 livre qu'on peut affirmer que la relation entre l'or et l'argent est de 1 à 15.

Ainsi, le système des étalons parallèles pourrait se définir un système dans lequel l'or et l'argent font office de métaux monétaires et d'étalons de mesure des valeurs, sans qu'il y ait entre eux une relation rigide. Ce système avait été faussé sous le régime français par l'introduction de la monnaie de carte et la mise en circulation d'une quantité effarante de papiers de dénominations diverses : lettre de change, billet du trésor, ordonnance, etc. Cependant, dès la prise de Québec, on assiste graduellement au retour de la circulation des espèces métalliques. En effet, l'ordonnance du roi de France qui suspend le paiement des lettres de change discrédite toute espèce de monnaie de papier. ${ }^{3}$ Les fournisseurs des armées françaises ne peuvent plus se procurer du blé qu'en échange d'espèces métalliques.

Comme il n'existe pas d'Hôtel des monnaies dans la province, on peut se demander d'où provient le numéraire. Essentiellement de trois sources. La première serait le gouvernement anglais qui paie en espèces sonnantes la solde et l'approvisionnement des troupes, le salaire des ouvriers et les émoluments des fonctionnaires. Le vainqueur amène donc dans ses goussets un numéraire peu connu avant la conquête dont la monnaie de compte s'appelle livre sterling et dont les pièces les plus courantes sont la guinée d'Angleterre, le chelin, le souverain. Le commerce international, axé désormais sur l'Angleterre, les colonies américaines et les antilles anglaises, constitue la deuxième source d'approvisionnement en numéraire. L'Amérique du Nord a une balance commerciale favorable avec les Antilles où circule la

3 Ord. du Roi, 15 oct. 1759, Doc. rel. à la monnaie, II : 928ss. 
monnaie espagnole dont la piastre est l'étalon. De ce fait, la monnaie espagnole abonde dans les treize colonies américaines; la conquête, en intégrant le Québec dans cet axe commercial, annexe une province nouvelle au royaume de la piastre espagnole. Une troisième source s'alimente au bas de laine de l'habitant. Ce dernier a souvent thésaurisé sous le régime français la monnaie métallique qui lui tombait sous la main. Ainsi, quand les troupes de Montcalm arrivent en 1755, elles apportent de beaux écus neufs que la population a soin de thésauriser. Cette monnaie française réapparaît à la fin des hostilités. L'ancien cours monétaire de la Nouvelle-France demeure donc toujours vivace: le louis d'argent, l'écu blanc, le double louis d'or, le liard perpétuent la présence française en Amérique. ${ }^{*}$ Dans la province de Québec circulent donc trois monnaies nationales qu'il faut ramener à une commune monnaie de compte pour faciliter les échanges, sinon on risque de poser aux habitants un puzzle indéchiffrable. En effet, sans une table d'équivalence, comment un habitant sur le marché de Québec pourrait-il coter le prix de ses denrées en trois monnaies différentes ou comment pourrait-il remettre la monnaie en espèces françaises à un client qui lui aurait payé sa marchandise en espèces anglaises ? La disparité de la monnaie pose donc aux administrateurs de la province un problème complexe, mais un problème moins ardu que la rareté du numéraire.

Cette situation n'est pas nouvelle. La pénurie des espèces sévissait à l'état endémique sous le régime français. C'est un phénomène ancien qui tend à s'accentuer avec la conquête. En effet, durant le régime militaire, certaines sources du numéraire menacent de se tarir. Dès 1765 , l'épargne et le capital de nombreux bourgeois et seigneurs seraient consumés, si on en croit les commerçants qui écrivent que «les nouveaux sujets ont été obligés de se deffaire a vil prix pour subsister de leurs argenteries, meubles et bijoux... L'argent que les nouveaux sujets envoient en france est dissipée; leur argenterie est au creuset $\gg .^{5}$

4 M. Trudel, Le Régime Militaire dans le Gouvernement des TroisRivières, 1760-1764:81ss. On y trouvera un exposé détaillé des principales pièces de monnaie qui ont cours dans la province.

5 Mémoire concernant les facultés de la province de Québec, mai 1765, $A S Q, \mathrm{Z2}-434-463$. 
Une diminution si marquée du capital s'expliquerait sans doute par la désorganisation de la production et du commerce consécutive à la guerre. En outre, le capital diminue tandis que le déficit dans la balance commerciale s'aggrave. Jadis, la situation n'était guère favorable. Depuis 1749 , le déficit se maintenait à un minimum de $300 \%$. Là originait la fuite du numéraire hors de la colonie. L'écart s'accroît davantage, selon les commerçants, durant les premières années du régime militaire: ne faut-il pas reconstituer les stocks ? partager avec les traiteurs d'Orange le commerce des fourrures ? reconstruire les agglomérations détruites ? importer des céréales et farines que ne fournissent plus des emblavures réduites ? Seule une balance des paiements favorable entre la colonie et la métropole pourrait enrayer la disette monétaire. Sous le régime français, les dépenses royales, de l'ordre de 3,000,000 livres tournois vers 1750 , comblent en partie le déficit commercial et répandent de la monnaie dans la colonie. La conquête ne change rien à ce mécanisme. Les commerçants admettent, en 1765, que «le commerce ne s'y est soutenu que par l'argent que les armées angloises et françoises y ont laissé, par celuy que les nouveaux sujets avoient en france ». ${ }^{6}$ Cependant, durant le régime militaire, les dépenses de la métropole anglaise dans la province de Québec moins considérables que celles de l'ancienne métropole française laissent subsister une balance des paiements défavorable à la colonie. Aussi «l'argent que les armées angloises et françoises avoient semé ici n'est-il pas passé à Londres en majeure partie ? $\gg,{ }^{7}$ de sorte que le numéraire se fait rare dans la colonie. ${ }^{8}$ Le Gouverneur pouvait pallier à la pénurie du numéraire en incitant la métropole à ressusciter le chantier de construction navale, à entretenir une forte garnison et à stimuler l'exportation des bois et des céréales. C'est la politique que les commerçants suggèrent à Murray.

Ce dernier n'est pas au bout de ses peines. Le retour aux espèces métalliques pose un autre problème: la liquidation du

${ }^{6}$ Loc. cit.

7 Loc. cit.

$8 \mathrm{M}$. Trudel a constaté cette pénurie du numéraire dans la diminution du revenu des fabriques et des quêtes. M. Trudel, op. cit., $86 \mathrm{~s}$. 
papier monnaie émis sous le régime français. Le problème ne manque pas d'importance puisque l'inventaire de 1764 évalue à 16,7 millions de livres tournois la monnaie de papier en circulation dans la colonie. Quelle était la valeur exacte de ce papier ? devait-on le laisser circuler ? comment en obtenir le remboursement? ce sont là des questions qui ne permettaient pas de dérobade.



Murray n'est pas homme à se dérober. Il attaque le problème monétaire sur tous les fronts à la fois avec autant de fermeté qu'il avait combattu les armées françaises. Sur le chapitre de la monnaie de papier, il se montre intraitable. Il en parle comme d'un brigandage public. Dès novembre 1759 , il déclare de nulle valeur le papier monnaie et en défend expressément la circulation. ${ }^{9}$ Gage et Burton endossent sa politique. Burton menace même de «punir dans toute l'étendue de mon gouvernement ceux qui en imposeront à la crédulité des habitants et les forceront de se contenter de ce payement frauduleux $\gg .^{10}$ L'ordonnance de Murray accentue la méfiance de la population à l'égard du papier monnaie, mais n'arrête pas sa circulation. On espère malgré tout que le roi de France honorera en partie ses engagements. Cet espoir est entretenu par le traité de Paris qui annonce, à l'article LV, «que S.M.T. (très disposée à rendre à Chacun la Justice qui lui est légitimement due), a déclaré et déclare que les d. billets et Lettres de Change seront exactement paiés, d'après une liquidation faitte dans un tems convenable, selon la distance des lieux et la possibilité; en évitant néanmoins que les billets et lettres de change que les sujets françois pourroient avoir au moment de cette déclaration ne soient confondus avec les billets et lettres de change qui sont dans la possession des nouveaux sujets du Roi de Grande-Bretagne ».11 Il y a beaucoup d'imprécisions dans cet article qui demeure muet sur la date de la

9 Ord. de Murray, 23 nov. 1759, $R A C$, 1918, app. B : 3ss.

10 Ord. de Burton, 22 sept. 1760, RAC, 1918, app. B : 85.

11 Traité de Paris, Doc. Rel. à la monnaie, II : 972. 
liquidation, le taux du remboursement et qui établit une distinction entre sujets français et sujets anglais. C'est la porte ouverte à la spéculation. Les fournisseurs de Londres, François Rybot, Daniel Vialars, Brook Watson et autres, demandent à leurs agents du Canada d'acheter la monnaie de papier «à condition qu'ils pussent l'acquérir avec un escompte très considérable $\gg .{ }^{12}$ Ils comptent sur leur influence dans les cercles politiques pour obtenir du Roi de France un taux de remboursement élevé.

Bientôt les agents des métropolitains parcourent la province en quête de papier. On offre de 15 à $25 \%$ de sa valeur nominale. Plutôt que de risquer une perte totale, nombre de personnes s'en départissent. Terroux, l'agent de Rybot, en acquiert à lui seul pour 1,333,681 livres françaises. ${ }^{13}$ Murray s'inquiète de cette spéculation qui pourrait causer des pertes considérables à la population. Il conseille de ne pas vendre son papier à vil prix et d'attendre la décision du roi de France. ${ }^{14}$ Il ordonne, en 1763 et 1764, de dresser un inventaire complet de la monnaie de papier dans la colonie. On en recense pour une somme de 16,7 millions de livres françaises. Pour imposant qu'il soit, le chiffre indique que les spéculateurs ont déjà râflé une quantité énorme de papier. Dans le gouvernement de Trois-Rivières, il n'en reste plus qu'un million 600 livres, parce que, constate Haldimand, « les coureurs des costes ont échangé à vil prix ce papier contre des marchandises ».

En dépit des ordonnances, la spéculation va donc bon train. On mise sur des pressions diplomatiques pour forcer le Roi de France à rembourser. Sa décision est connue le 29 juin 1764. Elle surprend tout le monde: ${ }^{15}$

- les lettres de change acquises avant 1759 par leurs propriétaires actuels seront payées en entier.

- les lettres de change, déclarées et visées, tirées en 1760 pour la subsistance des armées seront payées en entier.

12 Murray à Halifax, 14 fév. 1764, ibid., 992ss.

13 M. Hamelin, Jacques Terroux et le Commerce entre 1760 et 1765. Thèse manuscrite, 1961 .

14 Murray à Halifax, 14 fév. 1764, Doc. Rel. à la monnaie, II: 992s.

15 Ibid., 1012s. 
- les autres lettres de change déclarées et visées ne seront remboursées qu'à moitié de leur valeur nominale.

- les autres sortes de papier seront payés à un quart de leur valeur.

C'est un tolle parmi les spéculateurs londoniens, d'autant plus que la France ne semble pas vouloir donner suite à ses propositions. Les négociations traînent en longueur. Le gouvernement anglais et les commerçants confient leurs griefs au Sieur Henry Seymour Conway qui rencontre le Comte de Guerchy, ambassadeur de la France à Londres. Les négociateurs signent une convention, le 29 mars 1766. ${ }^{16}$ La France s'engage à racheter les lettres de change à $50 \%$ de leur valeur nominale, les ordonnances et les cartes à $25 \%$. Cependant elle ne paiera pas comptant; elle signera aux propriétaires de papier une reconnaissance de dettes qui porte un intérêt de $41 / 2 \%$. Ces reconnaissances ne jouissent pas d'un grand crédit. En mai 1766, un stock important de ces reconnaissances est vendu à Londres à $74 \%$ de sa valeur. ${ }^{17}$ Puis, en 1771, on connaît la fin de cette tragique histoire: la France ne paiera pas les reconnaissances de dettes. On peut lire dans la Gazette du 3 juin 1771: "France has at length effected the great stroke of politics she has long been aiming at: the Government is become bankrupt, and the whole score of State debts is rubbed out $\gg .^{18}$

Il est difficile de désigner les bénéficiaires de cette liquidation, si on excepte la France. Par contre, on peut affirmer à coup sûr que Baptiste supporta les frais de l'opération. En vain Murray essaya-t-il de lui éviter ce malheur puisque la solution lui échappait. Il n'en est pas de même pour la disparité de la monnaie, question sur laquelle le Gouverneur dispose des pleins pouvoirs. Dès la prise de Québec, Murray use de son autorité pour y mettre de l'ordre. Le 23 novembre 1759, dans le but de prévenir toute contestation, il publie une table d'équivalence

16 On trouvera le texte de cette convention dans James Stevenson, The Card Money of Canada: 24-31.

17 James IStevenson, The Currency of Canada after the Capitulation: 3.

18 Ibid., 4. 
des monnaies courantes. La table donne la valeur nominale en livre française et en livre anglaise cours de Halifax des monnaies en circulation. ${ }^{19}$ Entre les deux systèmes, il établit la relation suivante: un chelin anglais égale 1-4-0 livre française. Sans doute était-ce la relation que le commerce international avait consacrée depuis longtemps, en tenant compte du poids d'or ou d'argent qui définissait la livre anglaise d'Halifax et la livre tournois. ${ }^{20}$

SOMMAIRE DES TABLES D'EQUIVALENCE DE 1759, 1764 ET 1777

\begin{tabular}{|c|c|c|c|c|c|c|c|c|}
\hline \multirow{2}{*}{ Monnaie d'argent } & \multicolumn{2}{|c|}{ Poids } & \multicolumn{3}{|c|}{ Valeur en liv. frse } & \multicolumn{3}{|c|}{ Valeur en liv. canadienne } \\
\hline & Gros & Grains & 1759 & 1764 & 1777 & 1759 & 1764 & 1777 \\
\hline Piastre esp & 1 & 12 & $6-0-0$ & $6-0-0$ & & $0-5-0$ & $0-6-0$ & $0-5-0$ \\
\hline Ecu de Frar & 19 & 4 & $6-0-0$ & $6-0-0$ & & $0-5-0$ & $0-6-8$ & $0-5-6$ \\
\hline $\begin{array}{l}\text { Pièce française } \\
\text { de } 4-10-0\end{array}$ & & & & & & & 6 & $0-4-2$ \\
\hline Chelin anglais & & & & & & & $0-1-4$ & $0-1-1$ \\
\hline Pistereen & & & & & & & $0-1-2$ & $0-1-0$ \\
\hline Monn & & & & & & & & \\
\hline Johannes & 18 & 6 & $96-0-0$ & $96-0-0$ & & 4- $0-0$ & $4-16-0$ & 4- $0-0$ \\
\hline Noydore & 6 & 20 & $36-0-0$ & $36-0-0$ & & $1-10-0$ & $1-16-0$ & $1-10-0$ \\
\hline Caroline & 5 & 17 & & & & & $1-10-0$ & \\
\hline Guinée & 5 & 4 & $27-0-0$ & $28-0-0$ & & $1-3-4$ & $1-8-0$ & $1-3-4$ \\
\hline Louis d'or & 5 & 3 & $24-0-0$ & $28-0-0$ & & $1-0-0$ & $1-8-0$ & $1-2-6$ \\
\hline $\begin{array}{l}\text { Pistole frar } \\
\text { ou espagno }\end{array}$ & 4 & & & $21-0-0$ & & $0-18-0$ & $1-1-0$ & $0-18-0$ \\
\hline out & & 4 & $21-10-0$ & $21-0-0$ & & $0-18-0$ & $1-1-0$ & $0-18-0$ \\
\hline
\end{tabular}

Par son ordonnance, Murray vise à faire d'une pierre deux coups. Il entend mettre de l'ordre dans les monnaies en circulation et pallier à la pénurie du numéraire. En effet, le cours qu'il adopte s'identifie au cours d'Halifax: il tarifie à cinq chelins la

19 Dans son ordonnance, Murray spécifie qu'il s'agit du cours sterling. Cependant une rapide comparaison avec l'ordonnance de 1777 qui établit officiellement le cours d'Halifax dans la province montre que Murray, en 1759, a proclamé le cours d'Halifax. Ce dernier cours peut se définir ainsi: 70 gros d'argent ou 4 gros et $13 \frac{1}{2}$ grains d'or égalent une livre anglaise cours de Halifax. La livre sterling égale environ 72.72 gros d'argent. La relation entre l'or et l'argent fixée par l'ordonnance de 1759 est d'environ 1 à 15.3. On notera aussi que l'écu de France et le louis d'or sont tarifés, en 1759 , à un taux légèrement inférieur à celui du cours d'Halifax.

20 Ord. de Murray, 23 nov. 1759, RAC, 1918, app. B: 3ss. 
piastre espagnole qui ne vaut que 0-4-6 livre sterling. Les autres pièces de monnaies sont surévaluées de la même façon par rapport à la livre sterling. Murray se conforme ainsi à la tradition qui enseigne que la majoration des monnaies est un remède efficace contre la pénurie du numéraire. Si au lieu d'augmenter le volume du numéraire on augmente sa valeur, on arrive, croit-on alors, au même but, à savoir l'augmentation des moyens de paiement. Aux prises avec le même problème, les anciennes colonies anglaises ont depuis longtemps majoré leurs monnaies. La piastre espagnole vaut 5 chelins cours du Massachusetts, 7 chelins et 6 pence cours de la Pennsylvanie, 8 chelins cours d'York, 6 chelins cours de la Nouvelle-Angleterre. Loin d'innover, Murray applique donc les principes de la politique monétaire traditionnelle.

Les expériences du régime français montraient que l'augmentation des monnaies ne pouvait conduire qu'à l'inondation de la colonie par une monnaie usée et rognée. Par contre, la table d'équivalence pourrait mettre de l'ordre dans une situation monétaire confuse, si elle était proclamée dans les trois gouvernements. Mais tel n'est pas le cas. Dès l'automne 1760, Amherst introduit le cours d'York suivant lequel la piastre espagnole vaut 8 chelins. $^{21}$ Il est naturel qu'Amherst veuille imposer le cours monétaire des fournisseurs de son armée et qu'il accepte les données de la géographie qui relient Montréal à New York par le Richelieu et le lac Champlain. Gage, dans le gouvernement des Trois-Rivières, adopte lui aussi le cours d'York. Cette politique monétaire régionale minimise les conséquences de l'ordonnance de Murray. Si au niveau de l'habitant la situation monétaire est clarifiée parce que chaque gouvernement possède son cours monétaire, il reste que la situation demeure confuse pour les commerçants et les administrateurs qui doivent utiliser tantôt le cours de Montréal (York) et tantôt le cours de Québec (Halifax). D'autant plus que la présence de deux cours monétaires dans la même province ouvre la porte à la spéculation. Il suffit que dans

21 Ordonnance d'Amherst, 22 sept. 1760, $R A C$, 1918: 22. Il annonce que les voituriers à la solde de son armée seront payés selon le cours d'York. Nous n'avons malheureusement pu trouver la table d'équivalence publiée par Amherst. 
l'un des deux systèmes on donne une valeur plus grande à une monnaie réelle pour que cette monnaie disparaisse complètement d'une région donnée. C'est le cas de la monnaie française tarifée à un taux trop bas par Amherst; elle passe dans le gouvernement de Québec provoquant dans celui de Montréal «la rareté de l'argent surtout de la petite monnaie ». A la demande des commerçants, Gage, le successeur d'Amherst, rend justice à la monnaie française en augmentant sa valeur nominale. ${ }^{22}$

L'autonomie des gouvernements militaires perpétue donc la confusion. Leur disparition va-t-elle permettre l'établissement d'un cours monétaire uniforme à travers la province ? C'est ce que croit Murray, qui, en septembre 1764, profite de la réorganisation administrative pour proclamer une table d'équivalence des monnaies et un cours monétaire nouveau. ${ }^{23}$ L'ordonnance décrète qu'à partir du premier janvier 1765 le chelin anglais évalué à 2 gros grains et 21 grains d'argent ou à 4.43 grains d'or sera l'étalon du système monétaire canadien. Désormais la piastre espagnole vaudra 6 chelins et l'écu de France 6 chelins et 8 pence cours canadien. Pourquoi Murray délaisse-t-il le cours d'Halifax qu'il avait adopté en 1759 en faveur du cours de la NouvelleAngleterre ? Probablement parce que le cours de la NouvelleAngleterre lui permettait de donner une résonnance anglaise à la livre française: Murray appelle chelin canadien ce que l'habitant appelle livre française. L'ordonnance de 1764 rebaptise officiellement le système français toujours vivace parmi la population. Les calculs s'en trouvent simplifiés d'autant et l'habitant peut s'y retrouver plus facilement.

Murray en profite pour faire plusieurs mises au point. Il évalue à sa juste valeur l'écu de France et le louis d'or qu'il avait dévalué en 1759, il défend expressément de couper les piastres espagnoles dont les fragments servent de menue monnaie, car cette habitude a donné lieu à des fraudes et des abus; il limite enfin à un chelin le cours libératoire de la monnaie de cuivre. Ce dernier article a pour but d'éviter que la province soit inondée

${ }^{22}$ Ord. de Gage, 20 juillet 1762, $R A C, 1918$, app. B: 58.

23 Ord. de Murray, 14 sept. 1764, Gazette de Québec, 4 oct. 1764. 
d'une monnaie de cuivre sans valeur intrinsèque. Non sans raison Murray craint que la valeur nominale très élevée qu'il a donnée à la monnaie de cuivre incite les commerçants étrangers à payer leurs importations avec cette menue monnaie peu prisée dans les autres pays. Cependant Murray n'avait pas prévu que les impératifs de la géographie et les structures commerciales opposeraient une ferme résistance à ce nouveau cours canadien. Certains commerçants Montréalais, en contact avec les NewYorkais, continuent d'utiliser le cours d'York qui simplifie leur comptabilité. Il en est ainsi jusqu'à la révolution américaine. La sympathie que des Montréalais témoignent alors aux rebelles durant l'invasion du Canada amène le successeur de Murray à reviser le cours monétaire canadien. Dans une ordonnance en date du 29 mars 1777, Carleton proclame la mise en vigueur dans la province de Québec du cours d'Halifax. ${ }^{24}$ Il abandonne l'ancien cours qui calquait celui de la Nouvelle-Angleterre, afin de marquer la rupture politique qui existe entre la colonie de Québec et les colonies américaines. ${ }^{25}$

\section{JEAN HAMELIN}

Université Laval, Québec.

24 Ord. de Carleton, 29 mars 1777. Ordinances for the Province of Quebec (2ième série) : 70. Dans le système d'Halifax, le chelin joue le rôle d'étalon. Il vaut 3 gros et 12 grains d'argent ou 5.48 grains d'or.

25 L'Auteur remercie les membres du Centre de recherches historiques de Québec qui ont bien voulu mettre leur fichier à sa disposition. 